\title{
242. On Products of Quasi-Perfect Maps
}

\author{
By Yoshio TANAKa
}

(Comm. by Kinjirô KunUGI, M. J. A., Dec. 12, 1970)

1. Introduction. The purpose of this paper is to give a necessary and sufficient condition for the product map to be a quasi-perfect map under the condition that the image of the product map is a sequential space. Namely we shall prove

Theorem 1. Let $f_{i}: X_{i} \rightarrow Y_{i}(i=1,2, \ldots)$ be quasi-perfect maps and $\prod_{i=1}^{\infty} Y_{i}$ a sequential space. Then the following properties are equivalent.

(a) $\prod_{i=1}^{\infty} f_{i}$ is a quasi-perfect map.

(b) $\prod_{i=1}^{\infty} X_{i}$ satisfies the condition $\left(\mathrm{C}_{1}\right)$ below;

$\left(\mathrm{C}_{1}\right)$ : If each $K_{i}$ is a closed countably compact subset of $X_{i}$, then $\prod_{i=1}^{\infty} K_{i}$ is countably compact in $\prod_{i=1}^{\infty} X_{i}$.

(c) $\prod_{i=1}^{\infty} f_{i}^{-1}\left(C_{i}\right)$ is countably compact in $\prod_{i=1}^{\infty} X_{i}$ for each convergent sequence $C_{i}$ in $Y_{i}$. Here, by a convergent sequence we mean the union of the sequence and its limit point.

According to S. P. Franklin [4], a space $X$ is called a sequential space if a subset $F$ of $X$ is closed whenever $F \cap C$ is closed for every compact metric subset $C$ of $X$, and such a space is precisely the quotient of a metric space.

First countable spaces are sequential. Of course, sequential spaces are $k$-spaces. But the converse does not hold. Indeed, the Stone-Cěch compactification of a normal and non-compact space is not sequential.

We assume all maps are continuous and onto, and all spaces are $T_{2}$.

2. Proof of Theorem 1. First of all, we consider the following condition $\left(\mathrm{C}_{0}\right)$ on a space $X$.

$\left(\mathrm{C}_{0}\right)$ : Each sequence whose closure is countably compact has a sub-

The condition $\left(\mathrm{k}_{0}\right)$ in $\mathrm{T}$. Chiba [2] implies the condition $\left(\mathrm{C}_{0}\right)$, and the converse is true in regular $q$-spaces in the sense of E. Michael [7].

A space $X$ is called isocompact by $P$. Bacon [1] if every closed countably compact is compact.

Lemma 1. K-spaces, locally isocompact spaces, sequentially compact spaces, and regular spaces whose each point is a $G_{\delta}$-set all satisfy the condition $\left(\mathrm{C}_{0}\right)$.

Proof. Let $X$ be a $k$-space and $\left\{x_{i} ; i \in N\right\}$ a sequence in $X$ whose closure is countably compact. We assume all points $x_{i}$ are distinct. 
Let $x_{0}$ be an accumulation point of the set $\left\{x_{i} ; i \in N\right\}$. Then $\left\{x_{i} ; i \in N\right\}$ $-\left\{x_{0}\right\}$ is not closed. Hence there exists a compact subset $K$ of $X$ such that $K \cap\left(\left\{x_{i} ; i \in N\right\}-\left\{x_{0}\right\}\right)$ is not closed, which implies the infinite subset $K \cap\left(\left\{x_{i} ; i \in N\right\}-\left\{x_{0}\right\}\right)$ has a compact closure. Since a regular countably compact whose each point set is a $G_{i}$-set is first countable, a regular space whose each point is a $G_{\delta}$-set satisfies the condition $\left(\mathrm{C}_{0}\right)$. Since the proof for the other cases is easy, it is omitted.

By the Cantor "diagonal process" (for example, see p. 231 in J. Dugundji [3]), we have

Lemma 2. If each $X_{i}$ satisfies the condition $\left(\mathrm{C}_{0}\right)$, then $\prod_{i=1}^{\infty} X_{i}$ satisfies the condition $\left(\mathrm{C}_{1}\right)$.

Proof of Theorem 1. (a) $\rightarrow$ (b) : Let $K_{i}$ be closed countably compact in $X_{i}$. Then $f_{i}\left(K_{i}\right)$ is closed countably compact in $X_{i}$. Since sequential spaces are $k$-spaces, from Lemma 1 , each $Y_{i}$ satisfies the condition $\left(\mathrm{C}_{0}\right)$. Hence $\prod_{i=1}^{\infty} f_{i}\left(K_{i}\right)$ is countably compact by Lemma 2 . Since $\prod_{i=1}^{\infty} K_{i} \subset\left(\prod_{i=1}^{\infty} f_{i}\right)^{-1}\left(\prod_{i=1}^{\infty} f_{i}\left(K_{i}\right)\right)$ and $\left(\prod_{i=1}^{\infty} f_{i}\right)^{-1}\left(\prod_{i=1}^{\infty} f_{i}\left(K_{i}\right)\right)$ is countably compact by the quasi-perfectness of $\prod_{i=1}^{\infty} f_{i}, \prod_{i=1}^{\infty} K_{i}$ is countably compact.

(b) $\rightarrow$ (c) : Since each convergent sequence $C_{i}$ in $Y_{i}$ is compact and $f_{i}$ is quasi-perfect, $f_{i}^{-1}\left(C_{i}\right)$ is closed countably compact. Hence $\prod_{i=1}^{\infty} f_{i}^{-1}\left(C_{i}\right)$ is countably compact for each convergent sequence $C_{i}$ in $Y_{i}$ by (b).

(c) $\rightarrow$ (a): Let $F$ be a closed subset of $\prod_{i=1}^{\infty} X_{i}$. We shall prove $\left(\prod_{i=1}^{\infty} f_{i}\right)(F)$ is closed. Suppose $\left(\prod_{i=1}^{\infty} f_{i}\right)(F)$ is not closed. Since $\prod_{i=1}^{\infty} Y_{i}$ is sequential, from S. P. Franklin [4; Proposition 1.1], there exists a sequence $\left\{y_{n} ; n \in N\right\}$ such that $y_{n}=\left(y_{i n}\right) \in\left(\prod_{i=1}^{\infty} f_{i}\right)(F)$, that is, $\prod_{i=1}^{\infty}\left(f_{i}^{-1}\left(y_{i n}\right)\right) \cap F \neq \phi$, and $\left\{y_{n} ; n \in N\right\}$ converges to a point $p=\left(p_{i}\right)$ $\notin\left(\prod_{i=1}^{\infty} f_{i}\right)(F)$. Let $A_{i n}=\left\{y_{i j} ; j \geqq n\right\} \cup\left\{p_{i}\right\}$, and $F_{n}=\prod_{i=1}^{\infty}\left(f_{i}^{-1}\left(A_{i n}\right)\right) \cap F$. Then each sequence $A_{i n}$ is a convergent sequence. Hence $\prod_{i=1}^{\infty}\left(f_{i}^{-1}\left(A_{i n}\right)\right)$ is closed countably compact by (c). Moreover $F_{n} \supset F_{n+1} \neq \phi$ for each $n \in N$. Therefore $\bigcap_{n=1}^{\infty} F_{n} \neq \phi$, which implies $\prod_{i=1}^{\infty}\left(f_{i}^{-1}\left(p_{i}\right)\right) \cap F \neq \phi$. But this is impossible. Thus $\prod_{i=1}^{\infty} f_{i}$ is closed map. Since $\left(\prod_{i=1}^{\infty} f_{i}\right)^{-1}(y)$ is countably compact for each point $y$ of $\prod_{i=1}^{\infty} Y_{i}, \prod_{i=1}^{\infty} f_{i}$ is a quasi$k$-space.

Remark. (i): From the above proof, we see that a map of $X$ onto a sequential space $Y$ is quasi-perfect if and only if $f^{-1}(C)$ is countably compact for each convergent sequence $C$ in $Y$. From this and S. Hanai [5; Corollary 1.7], a space $X$ is countably compact if and only if the projection $P: X \times Y \rightarrow Y$ is closed for every sequential space $Y$.

(ii): The product map of quasi-perfect maps need not be closed and not even be quotient. 
As for the former, let $f:[0, \Omega) \rightarrow\{0\}$ and $i$ be the identity of $[0, \Omega]$, where $\Omega$ is the first uncountable ordinal number. Then $f \times i$ is not closed. As for the latter, let $X$ be the product of $[0, \Omega)$ with itself, and $Y$ be the space obtained from $X$ by identifying all points of the diagonal set of $X$, and let $f$ be the quotient map of $X$ onto $Y$. Then $f$ is quasi-perfect, but it is not a bi-quotient map defined by E. Michael [8]. From E. Michael [8; Theorem 1.3], $f \times i_{Z}$ is not quotient for some paracompact space $Z$.

3. Applications of Theorem 1. K. Morita [9] has introduced the notion of $M$-spaces and proved a space $X$ is an $M$-space if and only if there exist a metric space $Y$ and a quasi-perfect map of $X$ onto $Y$. Then, from Theorem 1 , we have

Corollary 1. Let $\prod_{i=1}^{\infty} X_{i}$ satisfy the condition $\left(\mathrm{C}_{1}\right)$, and each $X_{i}$ be an $M$-space. Then $\prod_{i=1}^{\infty} X_{i}$ is an $M$-space.

From Theorem 1 and Lemma 2, we have

Corollary 2. Let $f_{i}: X_{i} \rightarrow Y_{i}(i=1,2, \ldots)$ be quasi-perfect maps. If each $X_{i}$ satisfies the condition $\left(C_{0}\right)$, and $Y_{i}$ is first countable for each $i \in N$, then $\prod_{i=1}^{\infty} f_{i}$ is a quasi-perfect map.

Corollary 3. Let $f_{i}: X_{i} \rightarrow Y_{i}(i=1,2, \ldots)$ be closed, $X_{i}$ normal, and $\prod_{i=1}^{\infty} X_{i}$ sequential. Then $\prod_{i=1}^{\infty} f_{i}$ is quotient if and only if $\prod_{i=1}^{\infty} Y_{i}$ is sequential.

Proof. The "only if" part is obvious, for the quotient of a sequential space is sequential.

“if" ; Since $\prod_{i=1}^{\infty} Y_{i}$ is sequential and $C \subset \prod_{i=1}^{\infty} P_{i}(C)$ for every subset $C$ of $\prod_{i=1}^{\infty} Y_{i}$, where $P_{i}$ is the projection of $\prod_{i=1}^{\infty} Y_{i}$ onto $Y_{i}$, a subset $F$ of $\prod_{i=1}^{\infty} Y_{i}$ is closed whenever $F \cap \prod_{i=1}^{\infty} C_{i}$ is closed for every subset $\prod_{i=1}^{\infty} C_{i}$ of $\prod_{i=1}^{\infty} Y_{i}$, where each $C_{i}$ is a compact metric subset of $Y_{i}$. Hence, $\prod_{i=1}^{\infty} f_{i}$ is quotient whenever $\prod_{i=1}^{\infty}\left(f_{i} \mid f_{i}^{-1}\left(C_{i}\right)\right)$ is quotient for every compact metric subset $C_{i}$ of $Y_{i}$. We can then assume $Y_{i}$ are first countable. From K. Morita and S. Hanai [9], there exists $g_{i}: A_{i} \rightarrow Y_{i}$ such that $g_{i}$ is quasi-perfect, $A_{i}$ is a closed subset of $X_{i}$. Since $\prod_{i=1}^{\infty} A_{i}$ satisfies the condition $\left(\mathrm{C}_{1}\right)$, from Theorem $1, \prod_{i=1}^{\infty} g_{i}$ $=\prod_{i=1}^{\infty} f_{i} \cdot \prod_{i=1}^{\infty} i_{A_{i}}$ is quotient, which implies that $\prod_{i=1}^{\infty} f_{i}$ is quotient.

Remark. Even if $Y_{1} \times Y_{2}$ is separable metric, $f_{1} \times f_{2}$ need not be closed. Indeed, let $f:[0,1) \rightarrow\{0\}$ and $i$ be the identity of $[0,1]$. Then $f \times i$ is not closed.

Now, we consider the product of closed map.

Lemma 3. Let $f_{i}: X_{i} \rightarrow Y_{i}(i=1,2)$ be closed maps, $Y_{i}$ nondiscrete, sequential spaces, and $f_{1} \times f_{2}$ closed. Then $f_{1}$ and $f_{2}$ are quasiperfect maps.

Proof. Since $Y_{1}$ is a non-discrete sequential space, there exists a point $y_{1}$ which, considered as a subset, is not open in some metric sub- 
set $C$ of $Y_{1}$. As $f_{1} \times f_{2}$ is closed, $\left(f_{1} \mid f_{1}^{-1}(C)\right) \times f_{2}$ is also closed. Since $f_{1}^{-1}\left(y_{1}\right)$ is a $G_{\tilde{\delta}}$-set of $f_{1}^{-1}(C)$, from the proof given by T. Ishii [6; Theorem 2.3], we see that if we suppose $f_{2}^{-1}\left(y_{2}\right)$ is not countably compact for some point $y_{2}$ of $Y_{2}$, then $\left(f_{1} \mid f_{1}^{-1}(C)\right) \times f_{2}$ is not closed. This is impossible. Therefore $f_{2}^{-1}\left(y_{2}\right)$ is countably compact for each point $y_{2}$ of $Y_{2}$. Since $f_{2}$ is closed, $f_{2}$ is a quasi-perfect map. Similarly, $f_{1}$ is a quasi-perfect map.

From Theorem 1 and Lemma 3, we have

Theorem 2. Let $f_{i}: X \rightarrow Y_{i}(i=1,2, \ldots)$ be closed maps, $Y_{i}$ nondiscrete spaces, $\prod_{i=1}^{\infty} Y_{i}$ a sequential space, and $\prod_{i=1}^{\infty} X_{i}$ satisfy the condition $\left(\mathrm{C}_{1}\right)$. Then the following properties are equivalent.

(a) $\prod_{i=1}^{\infty} f_{i}$ is a closed map.

(b) $\prod_{i=1}^{\infty} f_{i}$ is a quasi-perfect map.

(c) Each $f_{i}$ is a quasi-perfect map.

Theorem 3. Let $f_{i}: X_{i} \rightarrow Y_{i}(i=1,2)$ be closed maps, $X_{i}$ sequential $M$-spaces, and $Y_{i}$ non-discrete, normal spaces. Then $f_{1} \times f_{2}$ is closed if and only if $f_{1}$ and $f_{2}$ are quasi-perfect maps.

Proof. The "only if" part follows from Lemma 3.

“if”, From K. Morita [11; Theorem 2.2], $Y_{i}$ are $M$-spaces. While, $Y_{i}$ are sequential. Hence $Y_{1} \times Y_{2}$ is sequential by [12; Corollary 2.3]. Consequently, $f_{1} \times f_{2}$ is a quasi-perfect map by Theorem 1 .

\section{References}

[1] P. Bacon: The compactness of countably compact spaces. Pacific. J. Math., 32, 587-592 (1970).

[2] T. Chiba: On $q$-spaces. Proc. Japan Acad., 45, 453-456 (1969).

[3] J. Dugundji: Topology. Allyn and Bacon Inc. Boston (1967).

[4] S. P. Franklin: Spaces in which sequences suffice. Fund. Math., 57, 107114 (1965).

[5] S. Hanai: Inverse images of closed mappings. I. Proc. Japan Acad., 37, 298-301 (1961).

[6] T. Ishii: On product spaces and product mappings. J. Math. Soc. Japan, 18, 166-181 (1966).

[7] E. Michael: A note on closed maps and compact sets. Israel. J. Math., 2, 173-176 (1964).

[8] _- Bi-quotient maps and cartesian products of quotient maps. Ann. Inst. Fourier, Grenoble., 18 (2), 287-302 (1968).

[ 9 ] K. Morita and S. Hanai: Closed mappings and metric spaces. Proc. Japan Acad., 32, 10-14 (1956).

[10] K. Morita: Products of normal spaces with metric spaces. Math. Annalen., 154, 365-382 (1964).

[11] _-: Some properties of $M$-spaces. Proc. Japan Acad., 43, 869-872 (1967).

[12] Y. Tanaka: On quasi-k-spaces. Proc. Japan Acad., 46, 1074-1079 (1970). 